\title{
La modellazione numerica dei contesti vulcanici
}

Sara Taviani - LinQ - Laboratorio di Idrogeologia Numerica e Quantitativa - Università degli Studi RomaTRE

sara.taviani@uniroma3.it

Nella comunità scientifica internazionale è nota la peculiarità in termini idrogeologici dei contesti vulcanici, Singhal e Gupta (2010) dedicano un capitolo all'idrogeologia delle rocce vulcaniche all'interno del loro libro "Applied Hydrogeology of Fractured Rocks", edito da Springer.

In questa nota si intende cercare di capire quali siano le peculiarità idrogeologiche dei contesti vulcanici che in maggior misura pesino nella rappresentazione del ciclo idrogeologico tramite un modello numerico di simulazione.

La presenza di depositi di diversa natura è una caratteristica intrinseca a tutti i distretti vulcanici. Un esempio è rappresentato dai diversi Distretti vulcanici presenti nel Lazio, all'interno dei quali si trovano, in sequenza, lave, piroclastiti, scorie, depositi di attività freatomagmatica, ecc. Questi depositi sono caratterizzati da proprietà idrauliche assai diverse tra loro: in termini di permeabilità primaria e secondaria (rispettivamente per porosità e per fratturazione).

Le geometrie dei depositi vulcanici non sono mai regolari, ma sono legate alle particolari modalità deposizionali dei diversi prodotti; ad esempio, la messa in posto di una lava che si muove per effetto della forza di gravità verso il basso e rallentata dalla propria viscosità, è molto diversa da quella di una colata piroclastica (miscela di magma e gas), in cui l'energia in gioco e la spinta propulsiva sono fortissime. D'altra parte all'interno di uno stesso deposito di colata piroclastica vi possono essere forti differenze tra le facies prossimali e distali e analogamente si differenzieranno le proprietà idrauliche all'interno di uno stesso deposito. Le facies prossimali sono generalmente caratterizzate dalla presenza di grossi frammenti di pomici, scorie, generalmente permeabili per porosità, le facies distali invece sono caratterizzate da depositi a matrice fine e scarsamente permeabili.

Emerge dunque una caratteristica tipica dei depositi vulcanici, l'eterogeneità, di cui si occupa Eaton (2006), il quale presenta una prospettiva sull'integrazione dell'eterogeneità geologica nelle simulazioni numeriche di flusso e rileva l'importanza assunta dagli sviluppi nel campo geostatistico per risolvere il problema dell'assegnazione della conducibilità idraulica alle singole celle di un modello di flusso.

Nel considerare le particolarità delle rocce vulcaniche nell'ambito della modellizzazione numerica, un tema da affrontare è quello dell'identificazione dei limiti del volume che si è interessati a rappresentare, la base delle vulcaniti e il limite laterale tra le vulcaniti e le diverse rocce sedimentarie presenti. Nel caso del Complesso Vulcanico dei Colli Albani, la presenza di materiale argilloso plio-pleistocenico presente quasi ovunque, al di sotto delle vulcaniti, aiuta a isolare il volume di rocce di interesse. All'interno di questo sarà necessa- rio poi identificare le principali superfici di discontinuità che influenzano la circolazione sotterranea (Mazza et al. 2009).

La messa in posto di prodotti vulcanici avviene attraverso centri di emissione (condotti craterici) e fessure di emissione; inoltre si ha spesso la formazione di faglie sin o post-eruttive (legate alla tettonica sin o post-eruttiva). Questi elementi geologico- strutturali costituiscono delle discontinuità ed assumono una certa rilevanza nel momento in cui entrano a far parte della modellazione. Si tratta di elementi comuni ai diversi contesti vulcanici, il cui ruolo di vie preferenziali per il flusso o invece quali barriere alla circolazione sotterranea andrà di volta in volta considerato.

I sistemi vulcanici sono inoltre spesso associati alla presenza di campi geotermici, alla presenza di emissioni di gas o di fluidi idrotermali, in questi casi si dovrà dunque modellizzare il trasporto di calore. Baiocchi et al. 2013 presentano i risultati della modellazione numerica effettuata per la comprensione dell'acquifero multi falda dell'area geotermica di Viterbo, attraverso l'uso del codice agli elementi finiti FEFLOW (Diersch 2002). Anche il codice alle differenze finite MT3DMS (Zheng and Wang 1999) può essere utilizzato nella simulazione del flusso di calore; Hecht-Mendez et al. (2010) presentano una valutazione del codice MT3DMS per la simulazione del trasporto di calore in sistemi geotermali chiusi, nel caso di mezzi porosi.

La morfologia stessa dei depositi vulcanici facilita la formazione di laghi, tanto nel caso di vulcani o stratovulcani non attivi, in corrispondenza del condotto eruttivo (il Lago di Albano ad es.), quanto nel caso delle caldere, dove la depressione vulcano tettonica viene occupata dai laghi (il Lago di Bracciano ad es.) Mazza et al. in press.

Si tratta spesso di laghi molto profondi: il lago di Bracciano è profondo 165 metri ed ha un'estensione di $57 \mathrm{~km}^{2}$, mentre quello di Albano, sebbene molto più piccolo, con i suoi $6 \mathrm{~km}^{2}$, è profondo 170 metri, proprio a causa del fatto che è ospitato nella depressione morfologica del condotto eruttivo. Il Crater Lake, nello stato dell'Oregon è profondo ben 594 metri. Volendo in questa sede porre l'attenzione sulle peculiarità della modellazione in ambiente vulcanico, si considera la profondità dei laghi quale elemento importante; bisogna infatti comprendere dove avvenga lo scambio tra le acque del lago e le acque sotterranee, oltre a tenere in debita considerazione il ciclo idrologico lacustre.

In contesti vulcanici in cui vi siano presenti i laghi, la modellazione numerica dovrà considerare l'interscambio tra acque sotterranee e superficiali (rappresentate dai laghi). In questo senso il codice numerico di flusso MODFLOW (McDonald and Harbaugh, 1999) è stato via via aggiornato negli ultimi 
anni per poter integrare diversi pacchetti, tra cui il pacchetto Lake (LAK Merrritt and konikov, 2000) ed il pacchetto Streamflow Routing (SFR, Prudic et al. 2004, Niswonger and Prudic, 2004, Niswonger and Prudic, 2006).

Il pacchetto Lake simula il livello del lago in funzione dell'intero bilancio idrico, compresi i flussi sotterranei in entrata ed in uscita, gli afflussi ed i deflussi simulati con il pacchetto Streamflow Routing, le precipitazioni e l'evaporazione, nonché gli eventuali prelievi dal lago. Il pacchetto Streamflow Routing introduce, tra le altre cose, la possibilità di simulare la propagazione del flusso nei corsi d'acqua e di mettere in comunicazione i corsi d'acqua con i laghi o le zone umide, sia in entrata che in uscita (Feinstein, 2012).

Nella figura 1 è riportato il risultato di una simulazione effettuata con il software Groundwater Vistas (GWV; Rumbaugh and Rumbaugh 2004) per l'area vulcanica Sabatina; il lago di Bracciano è stato simulato con il Lake package, il fiume Arrone con il Streamflow Routing package. Lo scambio avviene da nord verso sud, dapprima dall'acquifero al lago e poi da questo verso l'acquifero. Nella cross section 2 si può osservare come le celle Streamflow Routing nella parte più alta non drenino la falda, evidenziando quindi come l'alveo sia sospeso nella prima parte mentre nella parte bassa si attiva il drenaggio dell'alveo e le celle Streamflow Routing restituiranno il valore del flusso all'interno del corso d'acqua presente nell'area di studio.

Studi volti alla comprensione dell'idrogeologia dei distretti vulcanici della regione Lazio sono presenti in letteratura, molti dei quali si prefiggono l'obiettivo di costituire uno strumento utile alla gestione della risorsa (Capelli et al. 2005); sarebbe auspicabile che tutta la conoscenza acquisita su un contesto così particolare e altamente popolato, potesse servire da base per la costruzione di modelli numerici di flusso che possano costituire uno strumento ancor più efficace di supporto alla gestione.

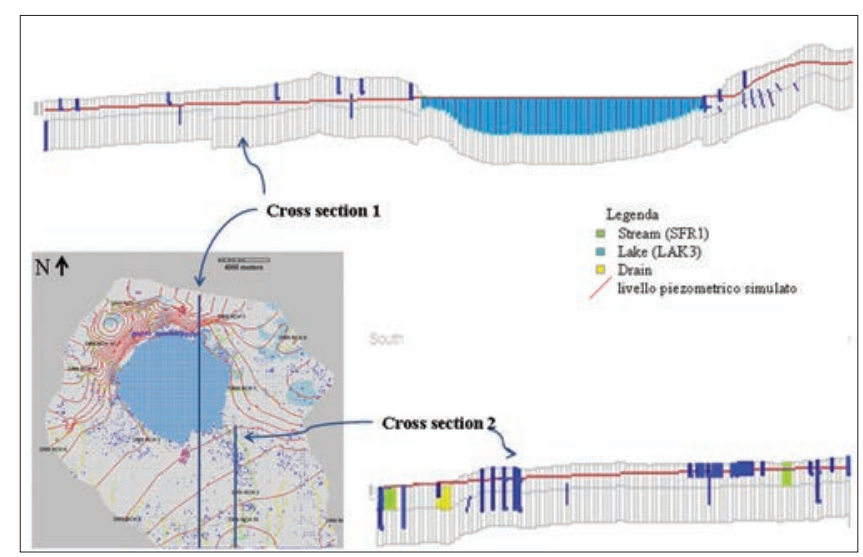

Fig. 1: Risultati di una simulazione numerica effettuati tramite Groundwater Vistas per l'area vulcanica Sabatina.

\section{BIBLIOGRAFIA}

Baiocchi A, Lotti F, Piscopo V (2013) Impact of groundwater withdrawals on the interaction of multi-layered aquifers in the Viterbo geothermal area (central Italy), Hydrogeology Journal 21, 13391353

Capelli G, Mazza R, Gazzetti C (2005) Strumenti e strategie per la tutela e uso compatibile della risorsa idrica nel Lazio. Gli acquiferi vulcanici. Quaderni di Tecniche di protezione ambientale. Protezione delle acque sotterranee 78. Pitagora, Bologna

Diersch HJG (2002) FEFLOW. Finite element subsurface flow and transport simulation system. Reference manual, WASY, Berlin

Eaton TT (2006) On the importance of geological heterogeneity for flow simulation, Sedimentary Geology 184, 187-201

Feinstein D (2012) Since "Groundwater and Surface Water-A Single Rosource": some U.S. Geological Survey advances in modeling groundwater/surface-water interactions. Acque sotterranee

Mazza R, Taviani S, Capelli G, De Benedetti AA, Giordano G, Quantitative hydrogeology of volcanic lakes: examples from the Central Italy Volcanic Lake District, Volcanic Lakes, Springer-IAVCEI Advances in Volcanology, in press

Mazza R, Capelli G, Taviani S, Teoli P, Gazzetti C, Rosa C, La Vigna F (2009) L’Unità idrogeologica dei Colli Albani: modellazione numerica degli acquiferi per un piano di uso compatibile della risorsa. In atti dei Lincei "La crisi dei sistemi idrici: approvvigionamento agro-industriale e civile" (22 marzo 2007 - Rome). Accademia Nazionale dei Lincei. Rome

McDonald MG, Harbaugh AW (1988) A modular three-dimensional finite-difference ground-water flow model. Techniques of WaterResources Investigations, Book 6. U.S. Geological Survey

Merritt ML, Konikow LF (2000) Documentation of a computer program to simulate lake-aquifer interaction using the MODFLOW ground-water flow model and the MOC3D solute-transport model: U.S. Geological Survey Water-Resources Investigations Report $00-4167,146 \mathrm{p}$

Niswonger RG, Prudic DE (2004) Modeling variably saturated flow using kinematic waves in MODFLOW: In Hogan, J.F., Phillips, F.M., and Scanlon, B.R., eds., Groundwater Recharge in a Desert Environment. American Geophysical Union (AGU), Water Science and Application 9, p. 101-112

Niswonger RG, Prudic DE (2006) Documentation of the Streamflow- Routing (SFR2) Package to include unsaturated flow beneath streams - A modification to SFR1: U.S. Geological Survey Techniques and Methods 6- A13, $48 \mathrm{p}$

Prudic DE, Konikow LF, Banta ER (2004) A new streamflow-routing (SFR1) package to simulate stream-aquifer interaction with MODFLOW- 2000: U.S. Geological Survey Open-File Report 2004$1042,95 \mathrm{p}$

Rumbaugh JO, Rumbaugh DB (2004) Guide to using groundwater vistas. Environmental Simulations, Reinholds, PA, USA

Singhal BBS, Gupta RP (2010) Applied Hydrogeology of Fractured Rocks, Springer

Zheng C, PP Wang (1999) MT3DMS: A modular threedimensional multi-species transport model for simulation of advection, dispersion and chemical reactions of contaminants in groundwater systems; Documentation and user's guide. U.S. Army Engineer Research and Development Center Contract Report SERDP-99-1, Vicksburg, Mississippi, 202 